\title{
Three-Dimensional Tetrathiafulvalene-Based Covalent Organic Frameworks for Tunable Electrical Conductivity
}

Hui Li, ${ }^{\dagger}$ Jianhong Chang, ${ }^{\dagger}$ Shanshan Li, ${ }^{\dagger}$ Xinyu Guan, ${ }^{\dagger}$ Daohao Li, ${ }^{\dagger}$ Cuiyan Li, ${ }^{\dagger}$ Lingxue Tang, ${ }^{\dagger}$ Ming Xue, ${ }^{\dagger}$ Yushan Yan, ${ }^{*}$ Valentin Valtchev, ${ }^{\dagger, l}$ Shilun Qiu $^{\dagger}$, and Qianrong Fang ${ }^{*, \dagger}$

${ }^{\dagger}$ State Key Laboratory of Inorganic Synthesis and Preparative Chemistry, Jilin University, Changchun 130012, P. R. China

${ }^{\star}$ Department of Chemical and Biomolecular Engineering, Center for Catalytic Science and Technology, University of Delaware, Newark, DE 19716, USA

${ }$ Normandie Univ, ENSICAEN, UNICAEN, CNRS, Laboratoire Catalyse et Spectrochimie, 6 Marechal Juin, 14050

Caen, France

\section{Supporting Information Placeholder}

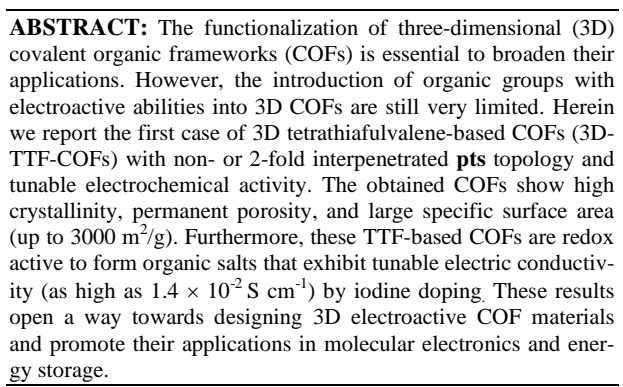

Covalent organic frameworks (COFs), as an exciting new type of crystalline porous polymers, are made from light elements, typically $\mathrm{H}, \mathrm{B}, \mathrm{C}, \mathrm{N}$ and $\mathrm{O}$, which crystallize into periodic networks by the formation of reversible covalent bonds. ${ }^{1-4}$ Owing to their high porosity, large specific surface area and good ther$\mathrm{mal} / \mathrm{chemical}$ stability, the COF materials have attracted much attention for considerable potential in gas storage and separation, 7 catalysis, ${ }^{8-12}$ organic electronics, ${ }^{13-16}$ and many others. ${ }^{17-23}$ Over the past decade, most of the reported work on COFs, however, were focused on two-dimensional (2D) COFs with layered eclipsed structures. Three-dimensional (3D) COFs, especially 3D functionalized COFs, have recently attracted extensive interest due to their unique porous features and excellent performances. ${ }^{24}$ ${ }^{29}$ We have synthesized a series of 3D functionalized COFs by the pre-synthesis or post-modification approach, including 3D Salphen-based COFs as catalytic antioxidants, ${ }^{30} 3 \mathrm{D}$ ionic liquidcontaining COFs for gas separation, ${ }^{31}$ 3D carboxy-functionalized COFs with selective extraction of lanthanide ions, ${ }^{32}$ and so on ${ }^{33-36}$ It must be noted, however, that the functionalization of 3D COFs remains largely unexplored, such as $3 \mathrm{D}$ electroactive COFs in particular. Thus, the development of new 3D functionalized COF materials is of critical importance for enriching the structural diversity and expanding their applications.
Scheme 1. Schematic representation of the strategy for preparing 3D-TTF-COFs ${ }^{a}$

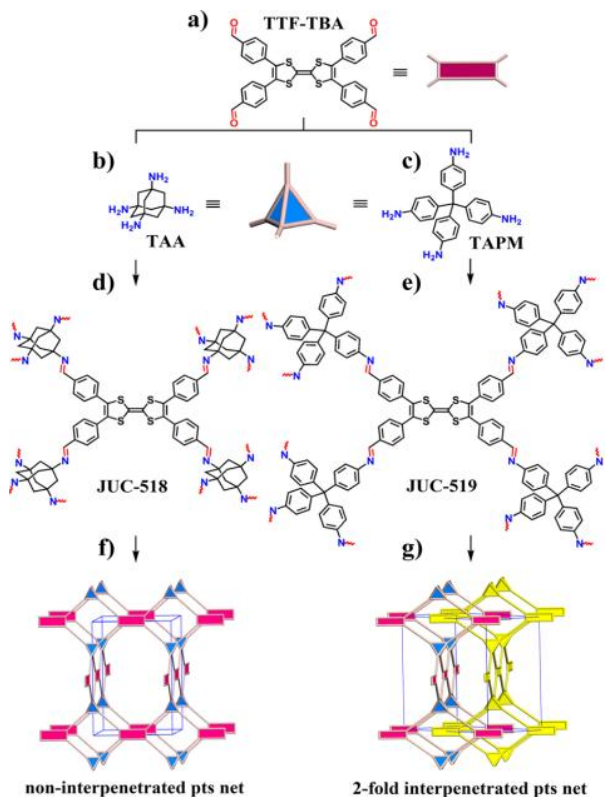

${ }^{a}$ Molecular structures of TTF (a) as a planar 4-connected building unit, TAA (b) as a short tetrahedral building unit, and TAPM (c) as a long tetrahedral building unit. 3D-TFF-COFs, denoted as JUC518 (d) and JUC-519 (e), are constructed by the condensation reaction of TTF and TAA or TAPM. Non- or 2-fold interpenetrated pts net in JUC-518 (f) and JUC-519 (g) 
Taking these considerations in mind, we report herein the first case of 3D electroactive COFs containing tetrathiafulvalene (TTF) functional units, denoted as 3D-TTF-COFs. Our results demonstrate that the obtained COFs have rare non- or 2-fold interpenetrated pts topology, high crystallinity and large surface area $(>$ $3000 \mathrm{~m}^{2} / \mathrm{g}$ ). More importantly, the TTF-based frameworks can be doped with electron acceptors (iodine, $\mathrm{I}_{2}$ ), and their conducting properties can be tuned from $1.8 \times 10^{-7}$ to $1.4 \times 10^{-2} \mathrm{~S} \mathrm{~m}^{-1}$ by the amount of $I_{2}$ and the temperature employed. To the best of our knowledge, this study is the first example of $3 \mathrm{D}$ COFs with noninterpenetrated pts topology and the application of 3D COFs as charge-transfer materials.
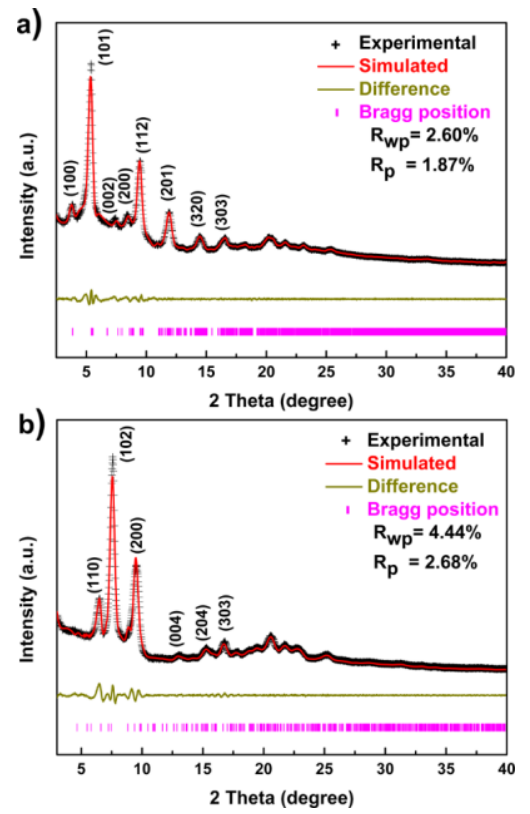

Figure 1. PXRD patterns of JUC-518 (a) and JUC-519 (b).

Our strategy for preparation of 3D electroactive COFs is based on a TTF derivative, tetrathiafulvalene-tetrabenzaldehyde (TTFTBA, Scheme 1a). As we all know, due to their excellent electrical activities, TTF or TTF-based derivatives have played an important role in the development of organic conducting materials. ${ }^{37}$

${ }^{39}$ Therefore, by the incorporation of TTF units into 3D porous $\mathrm{COFs}$, the resulting materials will not only promote electronic interactions between TTF molecules but also enable doping with electron acceptors (e.g., $\mathrm{I}_{2}$ ) to increase their conductivity. As shown in Scheme 1, TTF-TBA (Scheme 1a) was designed as a planar 4-connected building unit, and 1,3,5,7tetraaminoadamantane (TAA, Scheme 1b) or tetra(4aminophenyl)methane (TAPM, Scheme 1c) was chosen as a typical tetrahedral building unit. The condensation of TTF-TBA and TAA or TAPM produced 3D-TTF-COFs, termed as JUC-518 (JUC = Jilin University China, Scheme 1d) and JUC-519 (Scheme 1e). In light of the linking of tetrahedral and planar 4-connected building units, the structures of both COFs are expected to be the pts net (Scheme 1f and 1g). Since the TTF-TBA is connected by TAA as a short tetrahedral building unit $(\sim 3.1 \AA)$ and TAPM as a long tetrahedral building unit ( $5.9 \AA$ A, Figure S1), the resulting structures tend to be non- and 2-fold interpenetrated pts networks, respectively. ${ }^{40}$

a)

b)
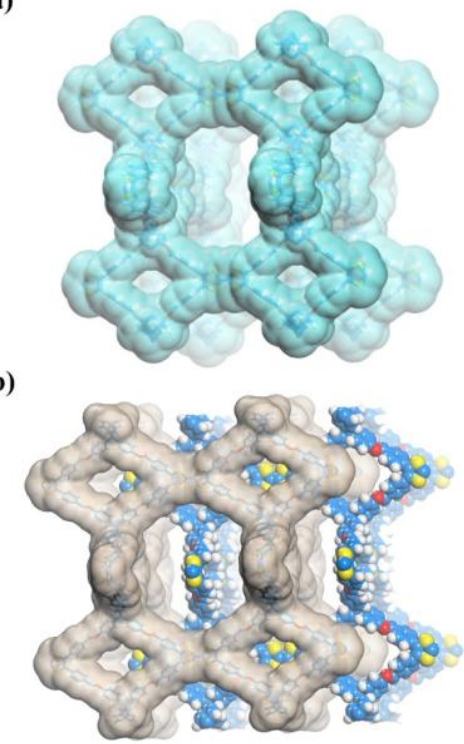

Figure 2. Extended structures of non-interpenetrated JUC-518 (a) and 2 -fold interpenetrated JUC-519 (b). C, blue; H, gray, N, red; S, yellow.

The synthesis of 3D-TTF-COFs was carried out by suspending TTF-TBA and TAA or TAPM in the mixed solvent of $o$ dicholobenzene $(o-\mathrm{DCB})$ and n-butanol $(\mathrm{n}-\mathrm{BuOH})$ in the presence of acetic acid followed by heating at $120{ }^{\circ} \mathrm{C}$ for 3 days. A variety of methods were employed for structural characterizations of 3DTTF-COFs. The morphology was examined by scanning electron microscopy (SEM) and transmission electron microscopy (TEM, Figures S2 and S3), which showed isometric crystals with a size of $0.2 \mu \mathrm{m}$. The Fourier transform infrared (FT-IR) spectrum exhibited a new peak at $1637 \mathrm{~cm}^{-1}$ for JUC-518 or $1625 \mathrm{~cm}^{-1}$ for JUC-519, which is a typical characteristic of $\mathrm{C}=\mathrm{N}$ bond. The concomitant disappearance of the $\mathrm{C}=\mathrm{O}$ stretching vibration (1701 $\mathrm{cm}^{-1}$ for TTF-TBA) and the N-H stretching vibration $\left(\sim 3324 \mathrm{~cm}^{-1}\right.$ for TAA and $\sim 3348 \mathrm{~cm}^{-1}$ for TAPM), confirmed the complete transformation of aldehyde and amine groups (Figures S4 and S5). The solid-state ${ }^{13} \mathrm{C}$ cross-polarization magic-angle-spinning (CP/MAS) NMR spectroscopy confirmed the presence of carbons from the imine groups by the peak at $154 \mathrm{ppm}$ for JUC-518 or 158 ppm for JUC-519 (Figures S6 and S7). Both 3D-TTF-COFs had high thermal stability $\left(\sim 400{ }^{\circ} \mathrm{C}\right)$ as shown by the thermogravimetric analysis (TGA, Figures S8 and S9), and can be preserved in different organic solvents and aqueous solutions of 1 $\mathrm{M} \mathrm{HCl}$ or $1 \mathrm{M} \mathrm{NaOH}$ (Figures S10 and S11).

The crystal structures of 3D-TTF-COFs were analyzed by the powder X-ray diffraction (PXRD) measurements in conjunction with structural simulations (Figure 1). After a geometrical energy minimization by the Materials Studio software package ${ }^{41}$ on the basis on non- or 2-fold interpenetrated pts net, their unit cell pa- 
rameters were acquired $(a=b=21.528 \AA, c=25.743 \AA$ and $\alpha=\beta$ $=\gamma=90^{\circ}$ for JUC-518; $a=b=18.673 \AA, c=30.679 \AA$ and $\alpha=\beta$ $=\gamma=90^{\circ}$ for JUC-519). Moreover, full profile pattern matching (Pawley) refinements were applied to their experimental PXRD patterns. Peaks at 3.78, 5.40, 7.43, 8.39, 9.44, 11.89, 14.36 and $16.57^{\circ}$ for JUC-518 belong to the (100), (101), (002), (200), (112), (201), (320) and (303) Bragg peaks of space group $\mathrm{P}_{2} / \mathrm{m}$ (No. 84); peaks at $6.51,7.45,9.48,12.99,15.19$ and $16.68^{\circ}$ for JUC-519 correspond to the (110), (102), (200), (004), (204) and (303) Bragg peaks of the space group $P 4_{2} / \mathrm{m}$ (No. 84). The refinement results can match well with the observed ones with good agreement factors $(R \mathrm{p}=1.87 \%$ and $\omega R \mathrm{p}=2.60 \%$ for JUC-518; $R \mathrm{p}=2.68 \%$ and $\omega R \mathrm{p}=4.44 \%$ for JUC-519). In addition, we tried alternative structures, such as 2-fold interpenetrated pts net for JUC-518 and non-interpenetrated pts net for JUC-519; however, their simulated PXRDs did not match to the experimental ones (Figures S12-15). In consideration of these results, the obtained COFs were proposed to have the expected frameworks with nonor 2-fold interpenetrated pts topology, which show microporous cavities with $\sim 1.62 \mathrm{~nm}$ for JUC-518 and $\sim 0.81 \mathrm{~nm}$ for JUC-519 (Figure 2). Remarkably, all the previous COFs with pts topology show interpenetrated structures and small dominated pores $(\sim 0.60$ $\mathrm{nm}){ }^{42-44}$ and thus the successful preparation of JUC-518 with non-interpenetrated nets will benefit the development of 3D COFs with large pores.

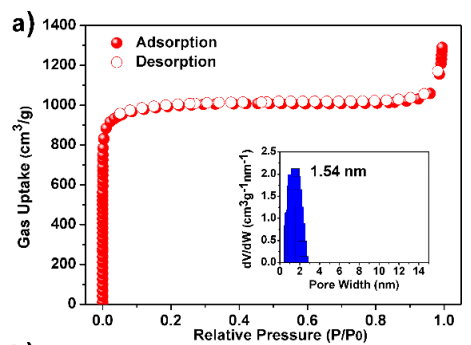

b)

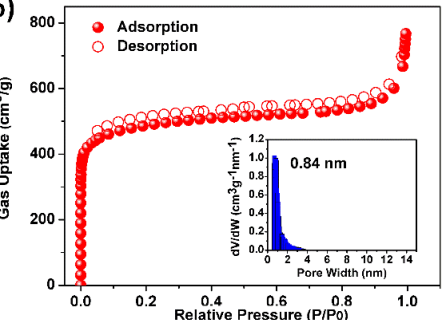

Figure 3. $\mathrm{N}_{2}$ adsorption-desorption isotherms for JUC-518 (a) and JUC-519 (b) at $77 \mathrm{~K}$. Inset: pore-size distribution calculated by fitting on the NLDFT model to the adsorption data.

The porosity and specific surface areas of 3D-TTF-COFs were determined by $\mathrm{N}_{2}$ adsorption analysis at $77 \mathrm{~K}$ (Figure 3 ). Both materials show a sharp uptake at a low pressure of $\mathrm{P} / \mathrm{P}_{0}<0.05$, which is a typical characteristic of the microporous material. The inclination of isotherms in the $0.9-1.0 \mathrm{P} / \mathrm{P}_{0}$ range and slight desorption hysteresis can be attributed to the presence of textural mesopores, which is from the agglomeration of COF crystals. ${ }^{3}$ The Brunauer-Emmett-Teller (BET) equation was carried out in the $0.05<\mathrm{P} / \mathrm{P}_{0}<0.30$ range of their isotherms, indicating BET surface areas of $3018 \mathrm{~m}^{2} \mathrm{~g}^{-1}$ for JUC-518 and $1513 \mathrm{~m}^{2} \mathrm{~g}^{-1}$ for JUC-519 (Figures S18 and S19). Obviously, owing to the non- interpenetrated structure of JUC-518, its BET surface area is much higher than those from all reported 3D COFs with the pts topology $\left(<1400 \mathrm{~m}^{2} \mathrm{~g}^{-1}\right) \cdot{ }^{42-44}$ Pore size distributions of 3D-TTFCOFs calculated based on the nonlocal density functional theory (NLDFT) demonstrated pores with the size of $1.54 \mathrm{~nm}$ for JUC518 and $0.84 \mathrm{~nm}$ for JUC-519 (Figure 3, inset), which are in good agreement with those of the proposed model $(1.62 \mathrm{~nm}$ for JUC518 and $0.81 \mathrm{~nm}$ for JUC-519).
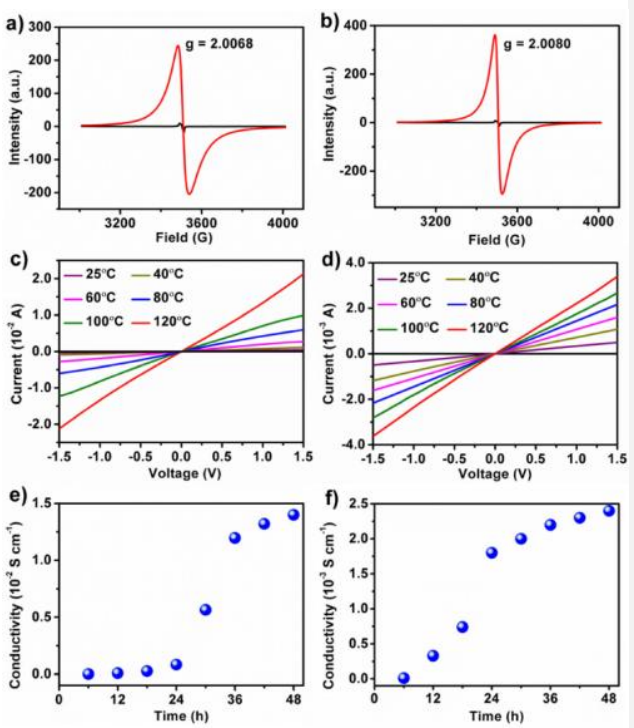

Figure 4. Solid-state EPR spectrum of JUC-518 (a) or JUC-509 (b) before (black) and after (red) $\mathrm{I}_{2}$ doping. Temperaturedependent I-V curves of JUC-518 (c) and JUC-509 (d) upon $\mathrm{I}_{2}$ oxidation (black curves: pristine $\mathrm{COFs}$ at $25^{\circ} \mathrm{C}$ ). Time-dependent electrical conductivities of JUC-518 (e) and JUC-509 (f) upon $\mathrm{I}_{2}$ oxidation.

Inspired by the abundant presence of TTF functional units, we firstly investigated the electrochemical behavior of 3D-TTF-COFs by cyclic voltammetry (CV). As shown in Figures S20 and S21, both COFs showed a reversible $\mathrm{CV}$ profile with two clear redox processes at 0.78 and $1.16 \mathrm{~V}$ for JUC-518 and 0.75 and $1.13 \mathrm{~V}$ for JUC-519 respectively, revealing that TTF units in COF frameworks retain their redox activities. Furthermore, the electrondonating nature of TTF units and permanent porosity of 3D-TTFCOFs could endow us to develop TTF-based charge-transfer (CT) materials. Both COFs were doped (oxidized) by exposure to $\mathrm{I}_{2}$ vapor. Figure $4 \mathrm{a}$ and $4 \mathrm{~b}$ showed electron paramagnetic resonance (EPR) spectra of JUC-518 and JUC-519 before and after $\mathrm{I}_{2}$ doping. Obviously, the pristine samples had very weak EPR spectra; however, the doped samples exhibited a significant paramagnetic intensity increased by approximately two orders of magnitude. After $\mathrm{I}_{2}$ doping, the sharp peaks were observed at $\mathrm{g}=2.0068$ for JUC518 and 2.0080 for JUC-519, clearly confirming the enhancing concentration of TTF radical cations. ${ }^{37-39}$ The PXRD patterns showed that 3D-TTF-COFs after $\mathrm{I}_{2}$ doping remained highly crystalline and structurally intact (Figures S16 and S17).

We further investigated the electrical conductivities of $\mathrm{I}_{2}$ doped 3D-TTF-COFs. Typically, the doped samples were compressed into cylindrical pellets with a diameter of $0.5 \mathrm{~cm}$ and a thickness 
of $0.2 \mathrm{~cm}$. The conductivities were conducted by using gold paste contacts in a two-probe configuration. As shown in Figures 4cand S22-37, the temperature dependences of the conductivities in the range 25 to $120^{\circ} \mathrm{C}$ revealed the semiconductor-like behavior of 3D-TTF-COFs after doping with $\mathrm{I}_{2}$. Furthermore, the conducting properties of 3D-TTF-COFs could be tuned with doping time from 6 to $48 \mathrm{hrs}$. These results are summarized in Tables S1 and S2. For example, conductivity of $2.9 \times 10^{-7} \mathrm{~S} \mathrm{~cm}^{-1}$ for JUC-518 at $25{ }^{\circ} \mathrm{C}$ is obtained after doping for $6 \mathrm{hrs}$, which could be increased to $2.7 \times 10^{-4} \mathrm{~S} \mathrm{~cm}^{-1}$ at $25^{\circ} \mathrm{C}$ upon extending the doping time to 48 hrs. Subsequently, the conductivity of JUC-518 could be extremely enhanced to $1.4 \times 10^{-2} \mathrm{~S} \mathrm{~cm}^{-1}$ by raising the temperature up to $120^{\circ} \mathrm{C}$. Notably, the electrical conductivities of 3D-TTF-COFs is higher than those from 2D TTF-based COF powders $\left(\sim 10^{-5} \mathrm{~S} \mathrm{~cm}\right.$ $\left.{ }^{1}\right) .45,46$ The high conductivities can be attributed to interconnected channels and high surface areas in 3D-TTF-COFs, in which TTF units can be fully oxidized into TTF radical cations.

In summary, we report 3D electroactive TTF-based COFs with non- or 2-fold interpenetrated pts topology, high crystallinity and large permanent porosity. Furthermore, these 3D-TTF-COFs are redox active to form organic salts that exhibit outstanding electric conductivity by doping $I_{2}$. This study not only develops a way towards designing 3D electroactive COF materials, but also promotes their applications in molecular electronics and energy storage.

\section{ASSOCIATED CONTENT}

\section{Supporting Information}

Methods and synthetic procedures, SEM, FTIR, solid state ${ }^{13} \mathrm{C}$ NMR, TGA, BET plots, and unit cell parameters. This material is available free of charge via the internet at http://pubs.acs.org.

\section{AUTHOR INFORMATION}

\section{Corresponding Author}

*qrfang@jlu.edu.cn

Notes

The authors declare no competing financial interests.

\section{ACKNOWLEDGMENT}

This work was supported by National Natural Science Foundation of China (21571079, 21621001, 21390394, 21571076, and 21571078), "111" project (B07016 and B17020), and the program for JLU Science and Technology Innovative Research Team. Q.F. and V.V. acknowledge the Thousand Talents program (China).

\section{REFERENCES}

(1) Côté, A. P.; Benin, A. I.; Ockwig, N. W.; O'Keeffe, M.; Matzger, A. J.; Yaghi, O. M. Porous, Crystalline, Covalent Organic Frameworks. Science 2005, 310, 1166.

(2) Ding, S. Y.; Wang, W. Covalent Organic Frameworks (COFs): from Design to Applications. Chem. Soc. Rev. 2013, 42, 548

(3) Huang, N.; Wang, P.; Jiang, D. L. Covalent Organic Frameworks: a Materials Platform for Structural and Functional Designs. Nat. Rev. Mater. 2016, $1,16068$.

(4) Diercks, C. S.; Yaghi, O. M. The Atom, the Molecule, and the Covalent Organic Framework. Science 2017, 355, 923.

(5) Kuhn, P.; Antonietti, M.; Thomas, A. Porous, Covalent Triazine Based Frameworks Prepared by Ionothermal Synthesis. Angew. Chem. Int Ed. 2008, 47,3450

(6) Han, S. S.; Furukawa, H.; Yaghi, O. M.; Goddard III, W. A. Covalent Organic Frameworks as Exceptional Hydrogen Storage Materials. $J$. Am. Chem. Soc. 2008, 130, 11580 .
(7) Wang, S.; Wang, Q.; Shao, P.; Han, Y.; Gao, X.; Ma, L.; Yuan, S.; Ma, X.; Zhou, J.; Feng, X.; Wang, B. Exfoliation of Covalent Organic Frameworks into Few-layer Redox-Active Nanosheets as Cathode Materials for Lithium-Ion Batteries. J. Am. Chem. Soc. 2017, 139, 4258.

(8) Ding, S. Y.; Gao, J.; Wang, Q.; Zhang, Y.; Song, W. G.; Su, C. Y.; Wang, W. Construction of Covalent Organic Framework for Catalysis: Pd/COF-LZU1 in Suzuki-Miyaura Coupling Reaction. J. Am. Chem. Soc. 2011, 133, 19816

(9) Vyas, V. S.; Haase, F.; Stegbauer, L.; Savasci, G.; Podjaski, F.; Ochsenfeld, C.; Lotsch, B. V. A Tunable Azine Covalent Organic Ochsenfeld, C.; Lotsch, B. V. A Tunable Azine Covalent Organic
Framework Platform for Visible Light-Induced Hydrogen Generation. Nat. Commun. 2015, 6, 8508.

(10) Peng, Y.; Hu, Z.; Gao, Y.; Yuan, D.; Kang, Z.; Qian, Y.; Yan, N.; Zhao, D. Synthesis of a Sulfonated Two-Dimensional Covalent Organic Framework as an Efficient Solid Acid Catalyst for Biobased Chemical Conversion. ChemSusChem 2015, 8, 3208.

(11) Wang, X.; Han, X.; Zhang, J.; Wu, X.; Liu, Y.; Cui, Y. Homochiral 2D Porous Covalent Organic Frameworks for Heterogeneous Asymmetric Catalysis. J. Am. Chem. Soc. 2016, 138, 12332 .

(12) Sun, Q.; Aguila, B.; Perman, J.; Nguyen, N.; Ma, S. Q. Flexibility Matters: Cooperative Active Sites in Covalent Organic Framework and Threaded Ionic Polymer. J. Am. Chem. Soc. 2016, 138, 15790.

(13) Wan, S.; Guo, J.; Kim, J.; Ihee, H.; Jiang, D. A Belt-Shaped, Blue Luminescent, and Semiconducting Covalent Organic Framework. Angew. Chem. Int. Ed. 2008, 47,8826

14) Bertrand, G. H. V.; Michaelis, V. K.; Ong, T. C.; Griffin, R. G.; Dinca, M. Thiophene-Based Covalent Organic Frameworks. Proc. Natl. Acad. Sci. USA 2013, 110, 4923.

(15) Dogru, M.; Handloser, M.; Auras, F.; Kunz, T.; Medina, D.; Hartschuh, A.; Knochel, P.; Bein, T. A Photoconductive Thienothiophene- Based Covalent Organic Framework Showing Charge Transfer Towards Included Fullerene. Angew. Chem., Int. Ed. 2013, 52, 2920.

(16) Du, Y.; Yang, H.; Whiteley, J. M.; Wan, S.; Jin, Y.; Lee, S. H.; Zhang, W. Ionic Covalent Organic Frameworks with Spiroborate Linkage. Angew. Chem. Int. Ed. 2016, 55, 1737.

(17) Oh, H.; Kalidindi, S. B.; Um, Y.; Bureekaew, S.; Schmid, R.; Fischer, R. A.; Hirscher, M. A Cryogenically Flexible Covalent Organic Framework for Efficient Hydrogen Isotope Separation by Quantum Sieving. Angew. Chem., Int. Ed. 2013, 52, 13219.

(18) Chandra, S.; Kundu, T.; Kandambeth, S.; BabaRao, R.; Arathe, M. Y.; Kunjir, S. M.; Banerjee, R. Phosphoric Acid Loaded Azo $(-\mathrm{N}=\mathrm{N}-)$ Based Covalent Organic Framework for Proton Conduction. J. Am. Chem. Soc. 2014, 136, 6570

(19) Zhou, T. Y.; Xu, S. Q.; Wen, Q.; Pang, Z. F.; Zhao, X. One-Step Construction of Two Different Kinds of Pores in a 2D Covalent Organic Framework. J. Am. Chem. Soc. 2014, 136, 15885 .

(20) Cai, S. L.; Zhang, Y. B.; Pun, A. B.; He, B.; Yang, J. H.; Toma, F. M.; Sharp, I. D.; Yaghi, O. M.; Fan, J.; Zheng, S. R.; Zhang, W. G.; Liu, $Y$. Tunable Electrical Conductivity in Oriented Thin Films of Tetrathiafulvalene-Based Covalent Organic Framework. Chem. Sci. 2014, 5,4693 .

(21) Jin, E. Q.; Asada, M.; Xu, Q.; Dalapati, S.; Addicoat, M. A.; Brady, M. A.; Xu, H.; Nakamura, T.; Heine, T.; Chen, Q. H.; Jiang, D. L. Two-Dimensional sp2 Carbon-Conjugated Covalent Organic Frameworks. Science 2017, 357, 673.

(22) Guo, Z. B.; Zhang, Y. Y.; Dong, Y.; Li, J.; Li, S. W.; Shao, P. P.; Feng, X.; Wang, B. Fast Ion Transport Pathway Provided by Polyethylene Glycol Confined in Covalent Organic Frameworks. J. Am. Chem. Soc. 2019, 141,1923

(23) Guan, X. Y.; Li, H.; Ma, Y. C.; Xue, M.; Fang, Q. R.; Yan, Y. S.; Valtchev, V.; Qiu, S. L. Chemically Stable Polyarylether-Based Covalent Organic Frameworks. Nat. Chem., 2019, 11, 587.

(24) El-Kaderi, H. M.; Hunt, J. R.; Mendoza-Cortes, J. L.; Côté, A. P.; Taylor, R. E.; O'Keeffe, M.; Yaghi, O. M. Designed Synthesis of 3D Covalent Organic Frameworks. Science 2007, 316, 268.

(25) Uribe-Romo, F. J.; Hunt, J. R.; Furukawa, H.; Klöck, C.; O'Keeffe, M.; Yaghi, O. M. A Crystalline Imine-Linked 3D Porous Covalent Organic Framework. J. Am. Chem. Soc., 2009, 131, 4570.

26) Bunck, D. N.; Dichtel, W. R. Internal Functionalization of Three- Dimensional Covalent Organic Frameworks. Angew. Chem. Int. Ed. 2012, 51,1885 .

(27) Beaudoin, D.; Maris, T.; Wuest, J. D. Constructing Monocrystalline Covalent Organic Networks by Polymerization. Nat. Chem. 2013, 5, 830 
(28) Liu, Y. Z.; Ma, Y. H.; Zhao, Y. B.; Sun, X. X.; Gándara, F.; Furukawa, H.; Liu, Z.; Zhu, H. Y.; Zhu, C. H.; Suenaga, K.; Oleynikov, P.; Alshammari, A. S.; Zhang, X.; Terasaki, O.; Yaghi, O. M. Weaving of Organic Threads into a Crystalline Covalent Organic Framework. Science 2016, 351,365

(29) Ma, T. Q.; Kapustin, E. A.; Yin, S. X.; Liang, L.; Zhou, Z. Y.; Niu, J.; Li, L. H.; Wang, Y. Y.; Su, J.; Li, J.; Wang, X. G.; Wang, W. D.; Wang, W. Sun, J. L. Y. Wrience 2017, 361, 48

(30) Yan, S. C.; Guan, X. Y.; Li, H.; Li, D. H.; Xue, M.; Yan, Y. S.; Valtchev, V.; Qiu, S. L.; Fang, Q. R. Three-dimensional Salphen-based Covalent-Organic Frameworks as Catalytic Antioxidants. J. Am. Chem. Soc. 2019, 141, 2920.

(31) Guan, X. Y.; Ma, Y. C.; Li, H.; Yusran, Y.; Xue, M.; Fang, Q. R. (31) Guan, X. Y.; Ma, Y. C.; Li, H.; Yusran, Y.; Xue, M.; Fang, Q. R.;
an, Y. S.; Valtchev, V.; Qiu, S. L. Fast, Ambient Temperature and Pressure Ionothermal Synthesis of Three-Dimensional Covalent Organic Frameworks. J. Am. Chem. Soc. 2018, 140, 4494.

(32) Lu, Q. Y.; Ma,Y. C.; Li, H.; Guan, X. Y.; Yusran, Y.; Xue, M.; Fang, Q. R.; Yan,Y. S.; Qiu, S. L.; Valtchev, V.; Postsynthetic Functionalization of Three- Dimensional Covalent Organic Framework for Selective Extraction of Lanthanide Ions. Angew. Chem. Int. Ed. 2018, 57,6042 .

(33) Fang, Q. R. Gu, S.; Zheng, J.; Zhuang, Z. B.; Qiu, S. L.; Yan, Y. S. 3D Microporous Base- Functionalized Covalent Organic Frameworks for Size- Selective Catalysis. Angew. Chem. Int. Ed. 2014, 53, 2878.

(34) Fang, Q. R.; J. Wang, H.; Gu, S.; Kaspar, R. B.; Zhuang, Z. B. (34) Fang, Q. R.; J. Wang, H.; Gu, S.; Kaspar, R. B.; Zhuang, Z. B.;
Zheng, J.; Guo, H. X.; Qiu, S. L.; Yan, Y. S. 3D Porous Crystalline Zheng, J.; Guo, H. X.; Qiu, S. L.; Yan, Y. S. 3D Porous Crystalline
Polyimide Covalent Organic Frameworks for Drug Delivery. J. Am. Chem Polyimide Covalent O
Soc. 2015, 137,8352 .

Soc. 2015, 137, 8352.
(35) Li, H.; Pan, Q. Y.; Ma, Y. C.; Guan, X. Y.; Xue, M.; Fang, Q. R.; Yan, Y. S.; Valtchev, V.; Qiu, S. L. Three-Dimensional Covalent Organic Frameworks with Dual Linkages for Bifunctional Cascade Catalysis. $J$. Am. Chem. Soc. 2016, 138, 14783.
(36) Li, Z. L.; Li, H.; Guan, X. Y.; Tang, J. J.; Yusran, Y.; Li, Z.; Xue, M.; Fang, Q. R.; Yan, Y. S.; Valtchev, V.; Qiu, S. L. Three-Dimensional Ionic Covalent Organic Frameworks for Rapid, Reversible, and Selective Ion Exchange. J. Am. Chem. Soc. 2017, 139, 17771.

(37) Farges, J. F. Organic Conductors: Fundamentals and Applications, Marcel Dekker, New York, 1994.

(38) Jérome, D. Organic Conductors: From Charge Density Wave TTF-TCNQ to Superconducting (TMTSF) ${ }_{2} \mathrm{PF}_{6}$. Chem. Rev. 2004, 104, 5565.

(39) Yamada, J. Sugimoto, T. TTF Chemistry: Fundamentals and Applications of Tetrathiafulvalene. Springer, Berlin, 2004.

(40) Bonneau, C.; Delgado-Friedrichs, O.; O'Keeffe, M.; Yaghi, O. M. Three-Periodic Nets and Tillings: Minimal Nets. Acta Crystallogr. 2004, A60, 517

41) Materials Studio ver. 7.0; Accelrys Inc.; San Diego, CA.

(42) Lin, G. Q.; Ding, H. M.; Yuan, D. Q.; Wang, B. S.; Wang, C. A Pyrene-Based, Fluorescent Three-Dimensional Covalent Organic Framework. J. Am. Chem. Soc. 2016, 138, 3302.

(43) Lin, G. Q.; Ding, H. M.; Chen, R. F.; Peng, Z. K.; Wang, B. S.; Wang, C. 3D Porphyrin-Based Covalent Organic Frameworks. J. Am. Chem. Soc. 2017, 139, 8705 .

(44) Ding, H. M; Li, J.; Xie, G. H.; Lin, G. Q.; Chen, R. F.; Peng, Z. K.; Yang, C. L.; Wang, B. S.; Sun, J. L.; Wang, C. An AIEgen-Based 3D Covalent Organic Framework for White Light-Emitting Diodes. Nat. Commun. 2018, 9, 5234.

(45) Ding, H. M.; Li,;Y. H.; Hu, H.; Sun, Y. M.; Wang, J. G.; Wang, C. X.; Wang, C. G.; Zhang, X. Wang, B. S.; Xu, W.; Zhang, D. Q A Tetrathiafulvalene- Based Electroactive Covalent Organic Framework. Chem. Eur. J. 2014, 20, 1461

(46) Jin, S. B.; Sakurai, T.; Kowalczyk, T.; Dalapati, S.; Xu, F.; Wei, H.; Chen, X.; Gao, J.; Seki, S.; Irle, S.; Jiang, D. L. Two- Dimensional Tetrathiafulvalene Covalent Organic Frameworks: Towards Latticed Conductive Organic Salts. Chem. Eur. J. 2014, 20, 14608. 
TOC Graphic: 